\title{
Masculinidades y Trabajo Social. Una aproximación teórica para nuevas intervenciones sociales y reivindicaciones profesionales.
}

\author{
Masculinities and Social Work. A theoretical approach for new social interventions and \\ professional demands.
}

Michael Muller Flury' (D)

\begin{abstract}
Resumen
Este artículo presenta una exposición teórica dirigida a abrir el camino para vincular la disciplina del Trabajo Social con la investigación y teorización de la masculinidad(es) y las implicaciones que ello puede (y debe) conllevar. Se ha basado la exposición teórica en una investigación del año 2018 sobre hombres que cuestionaban su masculinidad, añadiendo al enfoque inicial la visión de la intervención social, propia de nuestra profesión. Los conceptos tratados son la masculinidad hegemónica, las nuevas masculinidades y la masculinidad alternativa (en singular). Es preciso clarificar el origen y el significado de cada uno de los conceptos, así como explicar su llegada y desarrollo en el Estado español para que el Trabajo Social pueda entender los fenómenos que se dan entre las personas que atendemos y explicar, no justificar, ciertos comportamientos y actitudes, además de poder tomar consciencia de que no podemos reproducir ni validar los preceptos que emanan del sistema patriarcal y los roles de género. Como agentes de cambio, es imperativo que asumamos nuestro rol político para reclamar más formación en perspectiva de género y perspectiva feminista (en los grados universitarios de Trabajo Social), y más coeducación y feminismo en todos los niveles formativos de España. También debemos reclamar y aprovechar las ya existentes políticas de igualdad dirigidas a los hombres, para favorecer su autocuestionamiento y viaje hacia formas de masculinidad que renieguen y condenen firmemente los preceptos de la masculinidad tradicional.
\end{abstract}

Palabras clave: masculinidad hegemónica, nuevas masculinidades, masculinidad alternativa, Trabajo Social, intervención social.

\section{Abstract}

This article presents a theoretical exposition with the aim of up new way to link the discipline of Social Work to the investigation and theorization of Masculinity (s) and the implications that this can (and should) entail. The theoretical exposition made is based on a 2018 investigation where men questioned their masculinity, adding to the original focus a social intervention point of view as in our profession. The concepts discussed are hegemonic masculinity, new masculinities, and alternative masculinity (in the singular form). It is necessary to clarify the origin and meaning of each of these concepts, as well as to explain their arrival and development in the Spanish context so that Social Work can understand the phenomena that occur among the people we attend and explain, not justify, certain behaviours and attitudes, in addition to being able to become aware that we cannot reproduce or validate the precepts that emanate from the patriarchal system and gender roles. As agents of change, it is imperative that we assume our political role to demand more training in gender perspective and feminist perspective (in university degrees of Social Work), and more coeducation and feminism at all educational levels in Spain. We must also reclaim and take advantage of the already existing equality policies addressed at men, to favour their self-questioning and journey towards forms of masculinity that firmly deny and condemn the precepts of traditional masculinity.

Keywords: hegemonic masculinity, new masculinities, alternative masculinity, Social Work, social intervention.

Muller Flury, M. (2021). Masculinidades y Trabajo Social. Una aproximación teórica para nuevas intervenciones sociales y reivindicaciones profesionales. Itinerarios de Trabajo Social, 1, 23-30. https://doi.org/10.1344/its.voi1.32496

\section{Introducción.}

El presente artículo, de carácter teórico, aborda algunos conceptos que forman parte de la agenda social y que son utilizados en la literatura científica, pero que puede requieran de un análisis para conocer su origen, diferencias, matices y qué aportan e implican para la disciplina del Trabajo Social. La teoría utilizada proviene de una búsqueda intensa en diversas bases de datos, revistas científicas, trabajos académicos, etc. Cabe indicar desde el inicio que el presente artículo cuenta con la utilización de lenguaje inclusivo e incorpora el formato de citación propio de los Women's Studies ${ }^{2}$, respetando la normativa APA.

Los conceptos tratados son: masculinidad hegemónica, nuevas masculinidades y masculinidad alternativa (en singular). Se pretende dar respuesta a cómo nacieron, a paña en relación con dichos términos y sus implicaciones. Hay diversas investigaciones teóricas y publicaciones, como las desarrolladas por Jokin Azpiazu (2013, 2017), que han ido poniendo luz a lo que significa la masculinidad, así como los cambios observados en ella. El estudio qué hacen referencia y qué situación encontramos en Es-
ACEPTADO: 01/12/2020 PUBLICADO: 21/01/2021

${ }^{1}$ Trabajador social. Mediador comunitario. Ajuntament de Palma, Mallorca. 
de los hombres y las masculinidades es un campo diverso de interes que se ha estudiado desde diversas disc plinas, incluyendo la psicología, la sociología, la salu pública y otros (Joel Wong \& Angela Horn, 2016; Raewy Connell et al., 2005). Aśí, las masculinidades cuentan con
una agenda científica yacadémica, y con otra de acción social y politica. En el 2012 se podian encontrar 4133 re des" (Raewyn Connell, 2012). En el 2018, 9553 referencias provenientes de diversas revistas cientificas dedicadas Masculinities, del SAGE Journals, la revista Psychology of Men \& Masculinity (de la APA) o la más reciente Masculnidades y Cambio Social - Masculinities and Social Change,
publicada por Hipatia Press e indexada en bases internacionales.

Todo ello en un momento donde, gracias al peso que constitución identitaria del género en los hombres. Una realidad que se interrelaciona con la disciplina del Tra bajo Social para dar un primer paso en la construcción de nuevos marcos teóricos que permitan intervenciones sociales con hombres para perseguir el cambio en su ma nera de ser hombre. Tanto asi que se pueden encontra recursos existentes especificamente dirigidos a este "colectivo"

\section{Metodología.}

La información teórica que se expone en el artículo proviene de la búsqueda realizada en la literatura científica. Se utilizan, por tanto, fuentes secundarias como la litera tura cientifica teórica e investigaciones sobre la temática "rratada. Se buscaron terminos como "masculinidades", "masculinidad alternativa", "nuevas mascullididades", exponerse de forma resumida a lo largo del artículo. En cuanto a los aspectos éticos, se resumen en la correcta aplicación de las normas de citación para diferencia claramente las aportaciones del autor de las que perte necen a otros manuscritos, libros, artículos... Siguiendo este hilo, debe mencionarse la limitación existente el presente articulo. La investigación teórica se realizó años atrás y no tenia en aquel entonces un enfoque esp cificamente orientado hacia la práctica del Trabajo Sociá. Esto se ha corregido con una búsqueda de las palabras Cabe exponer que no se han encontrado, prácticamente, resultados de interés que vinculen estrechamente la te mática tratada con la profesión del Trabajo Social.

\section{Feminismo y masculinidad(es).}

No sería correcto hablar de la masculinidad o de los estudios críticos sobre masculinidades (iniciados en los años cual se nutren los elementos anteriores; el feminismo (Azpiazu, 2017; Ritxar Bacete, 2017). Se define como discurso político basado en la justicia, una teoría y una practica politica articulada por las mujeres para acab con las discriminaciones que sufren y para cambiar la soten identificar de una forma clara que el Trabajo Social también se ha nutrido del feminismo, llevando de una 政 tasión de acabar con las discriminaciones y consegur

(a) caracterizado como una unidad homogénea, amorfa y opresiva, qu gnización existente en torns a las relaciones. Una ordonde el eje principal de diferenciación es el sistema de género (Kate Millet, 1970). Este, a su vez, es entendido como un constructo social que analiza y/o explica desde a psicología y otras disciplinas, las identidades sexuales (Tania Serrano y Marcho Schirralli, 2009). Este sistema produce, según Daniel Gabarró (2011), la perpetuación del sexismo y la homofobia. Permite a los hombres tener I conflicto y a la ansiedad (Ghail Pheterson, 2013). ansiedad justificada por lad (Chall Pheterson, 2013). Una cia de los hombres (Michael Kaufman, 1999) o por las erribles cifras en el contexto español, donde 566 mujees fueron asesinadas por hombres entre el año 2000 y el 2008 (Dani Leal, 2008) y sólo en el año 2019, se produjeon 55 asesinatos machistas.

contexto anterior provoca que paulatinamente surjan los estudios de género en la investigación académica para 2005). Para algunos y algunas, la primad (Connell et al., 2005). Para algunos y algunas, la primera aparición de Sexe de Simone de Beauvoir (1949), donde se explicitaba que los hombres eran sometidos a mayores presiones en el proceso de socialización por el continuo refuerzo de la virilidad (Juan Carlos Peña, 2013). Para otras, se situaría en Sex and Temperament in Three Primitive Societies, 1935 (Margaret Mead), un estudio que ofrecía una visión plural de las masculinidades (Aharon Fernández, 2009). No es atrevido afirmar que los hombres llevan tiempo formando parte de la bibliografía feminista en calidad de miembros del patriarcado, pero poco tiempo siendo condición de género (Benno de Keijzer, 2003) Peña, 2013; Alvaro Ruiz, 2007; Serrano y Schirralli, 2009).

1. Masculinidad hegemónica.

Si para el mundo del Trabajo Social, Mary Richmond es na referente de las que aprender y leer, para muchos muchas la principal figura en haber teorizado sobre la masculinidad y el concepto de masculinidad hegemónica de Keijzer, 2003; Peña, 2013). El concepto forma parte del grueso de investigaciones en torno a las masculinidades, las cuales provienen mayoritariamente del mundo anglosajón (Men's Studies). Según Ruiz (2007), hacen falta más bre masculinidades lleguen a más población $Y$, añado deberían producirse en nuevos contextos para ampliar, punto de mira (estamentos sociales, culturas, países.) Existen 4 tipos de masculinidades (dentro del sistema de relaciones de género): hegemónica, cómplice, subordinada y marginada (Raewyn Connell, 1995). La autora define la hegemónica (también llamada "tradicional"), como aquella que legitima el patriarcado, garantiza la posición dominante de los hombres y la subordinación de las mujeres. Una masculinidad que tiene 4 mandatos para los hombres: no tener nada de mujer, ser importante, ser un hombre duro y enviar a todo el mundo infierno (Leal, 2008), que "ho es ni un bebé ni un homosexual" (Elisabeth Badinter, 1993, p. 55).

La masculinidad hegemónica se aprende en el proceso de socialización, un proceso que limita las posibilidades de pone pautas de comportamiento $y$ actitudes no que in asumibles como, por eremplo, llegar a ser un superhéree (Ander Bergara et al., 2008). Fomenta la competitividad la no expresión de sentmientos y vivir las relaciones sexuales como un examen de la virilidad (Dani Leal et al, 2010), asi como la valentía, la agresividad y la competitividad (Jose Manuel Salas y Alvaro Campos, 2001). Tien presencia en el mundo de la ficción, produciendo perso najes que, expuestos a los niños y las niñas (guerreros, hombres de negocio...), modelan su imagen de lo que escolar, aun habiendo avances en materia de coeduca ción, la masculinidad hegemónica sigue teniendo mucha presencia (Cecilia Azorín, 2017; Oriol Ríos, 2013). Hablamos por tanto de la construcción de una identidad social (actitudes, comportamientos, roles...) que convive con otras identidades del entorno.

Existen algunas críticas hacia el concepto de masculindad hegemónica, ya que pocos hombres practican rigu rosamente los patrones marcados. La mayoría se encuense aprovecha de los beneficios del dividendo patriace de una forma sutil (Bermúdez, 2013; Peña, 2013). Además, la definición sólo funciona en un contexto blanco y heterosexual, cuando las diferencias se producen a nive global (Àngel Carabí y Josep Armengol, 2015). La masculinidad hegemónica no puede entenderse por tanto como una visión única de la jerarquía y de las caracteristicas del género (Raewyn Connell \& James Messerschmidt, 2005), ni goza de total legitimidad debido una crisis en el orden de género (Connell, 1995). Dich cuestionamiento de la identidad masculina en un con texto de reflexín en torno a los cambios sociales y culturales (Chris Haywood \& Mairtin Mac an Chaill, 2003) de la acción del capitalismo (que saca la mano de obra femenina del ámbito doméstico para aprovisionar proceso de producción de bienes de consumo) (Salas Campos, 2001) y del avance del feminismo (Bacete, 2017; Sergi Chouciño, 2017; Joan Sanfélix, 2011). Una crisis que ha provocado un cambio real, diverso e nacabado en los hombres que se ha ido mate divizando con el nacimiento

3.2. Nuevas masculinidades.

Los grupos de hombres que configuran nuevas formas de ser hombres nacen mayoritariamente en paises anglosajones a partir de los años 70 (concretamente en los y se fueron extendiendo progresivante por Europa Latinoamérica (Joan M. Navarro y Miralles, 2002). Existen diversos movimientos; desde lo defen mos de la masculinidad hegemónica, hasta los que tie nen un carácter progresista, de cambio y de ruptura. Se resumen a continuación

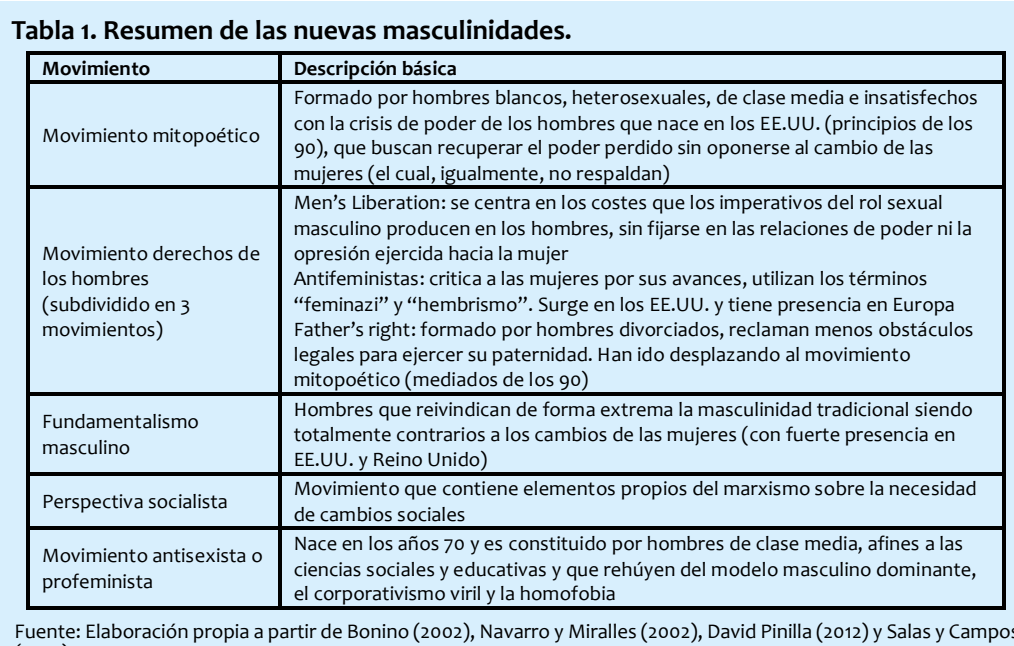

El Trabajo Social, como profesión que trabaja con personas socializadas en un contexto marcado por el géne Tanto si atiende a una mujer que ha sido maltratada por un hombre (para entender [no justificar], qué pensa acometer agresiones del tipo que sean) como si se atiende a un hombre agresor, para profundizar con él y abrir las puertas de la reflexión, conocer otras formas de ser hombre, desaprender lo aprendido.

De ahí que se sitúe el interés en el movimiento antise xista o profeminista, ya que se constituye, en el terren culinidad alternativa Por eso aquíse hacente de la mas rificadora de hablar de masculinidad alternativa en singlar, ya que hace referencia a aquella que se define como la propia de hombres activos en la lucha contra la violencia de género (junto con las mujeres), que rechazan personas con valores no igualitarios o violentas, que busca relaciones igualitarias basadas en el deseo y el amor (Ramón Flecha et al., 2013) y que reniegan de los principios 3.3. Masculinidad alternativa.

Se sitúa el inicio de la masculinidad alternativa (profemnista y/o antisexista) en el norte de Europa en grupos hombres que reflexionan y deconstruyen la masculinidad tradicional (Serrano y schirralli, 2009), recogiendo la poder y los privilegios de los hombres (Kaufman Es un movimiento construido con parámetros trans gresores que pasan por una mayor participación de los hombres en la esfera reproductiva, en el cuidado personal, emocional y de salud, así como el ejercicio de una paternidad presente en la atención de los y las menores (Carabi y Armengol, 2015; Ana Marín, 2014; Pinilla, 2012). Los hombres de este movimiento participan en proyectos de igualdad de género (Revi Karkara, 2007). Se responsabilizan del sexismo y buscan desertar el machismo te. Son plenamente conscientes de que la igualdad de género beneficia tanto a hombres como a mujeres (Ba cete, 2017) y, finalmente, son el reflejo de una realidad; masculinidad hegemónica resulta ser cada vez más difić 
de entender por los hombres contemporáneos (Saideh Garousi et al., 2016). Estos perciben la socialización masculina como una fuente de problemas personales y so-
ciales (Ruiz, 2007). Recoge dicho malestar la teoría del ciales (Ruiz, 2007). Recoge dicho malestar la teoría del
Gender Role Conflict (de ahora en adelante GRC), desarroGender Role Conflict (de ahora en adelan
Ilada por James O'Neil a partir del 1981.

Ilada por James ONell a partir del 1981. género tiene consecuencias negativas para la persona y su entorno, ya que son roles estrictos, sexistas y restrictivos, provocando estas restricción personal (James O’Neil et al., 2016). Son roles que deshumanizan a los hombres, mujeres e infancia, aunque los hombres suele negar que los roles les provoquen dificultades, ya que
hacerlo implica una amenaza a su status quo. Se ha utthacerlo implica una amenaza a su status quo. Se ha uti-
lizado la teoría GRC en investigaciones desde hace más lizado la teoría GRC en investigaciones desde hace más de 25 años (recopiladas por James O'Neil y Robyn Denke
en 2016 dentro del Handbook of Men and Masculinities). Los autores consideran que el CRC aparece con mas fuerza en edades menos avanzadas en hombres, y con el tiempo pueden transformarse en una motivación para cambiar y desafiar los roles de género asignados. Esta realidad nos presenta la oportunidad de entende la importancia de acercar la perspectiva feminista y de género a las aulas, implementar la coeducación a gran escala, para favorecer un cuestionamiento. Cuando los hombres aceptan la existencia del estrés de género y se cuestionan su masculinidad, estos pueden vivir un $\mathrm{mo}-\mathrm{s}$ movimientos intorna (Ruiz, 2007). proba de ello son los en el año 1991, cuando empezó la campaña del Lazo Bla co donde hombres quisieron mostrar públicamente rechazo hacia la violencia perpetrada hacia las mujeres (Christine Ricardo, 2015). También son prueba de ello las asociaciones internacionales como LASOM (Noruega) Les Traboules (Francia) (Bonino, 2002), o el movimien
He for She que lanzó la ONU en el 2014 (Bacete, 2017). He for She que lanzó la ONU en el 2014 (Bace

3.4. Masculinidad alternativa en España. va (profeminista y/o antisexista) a España? Aparece los años 80 (Serrano y Schirralli, 2009), y empieza a funcionar a partir de los años 9o. En el año 1988 el grupo de en contra de la violencia ejercida de hombres hacia mu jeres (a raíz del asesinato de Ana Orantes a mano de exmarido, diciembre de 1997). Poco después se celebra ron 2 congresos sobre "hombres e igualdad" en San Sebastián y Jerez, los cuales aumentaron el interés público sobre los grupos de hombres igualitarios (Bonino, 2002). Precisamente la Delegación de lgualdad y Salud del Ayun-s tamiento de Jerez lanzo un programa llamado "Hombres por igualdad", que es un referente a nivel internaciona. Otro programa destacable es el d'Emakunde, un progr coordina y evalúa políticas de igualdad realy e, activa entre hombres y mujeres (Emakunde - Instituto Vasco de Mujer, 2010). También encontramos la asociación AHIC (Asociación de Hombres por la Igualdad de Género) que nace en el 2001 en Málaga y tiene presencia estatal, ofr ciendo a los hombres formas de ser libres del mandato del machismo y el patriarcado (AHIGE, 2018). Su notoriedad le ha permitido tener voz y aparecer en un diario
como es El Confidencial (Boria Rodrigo, 2018). Asociacio- tes de reciente creación como Homes Transitant, de las Slas Baleares que emiten programa de radio en Ona $M$ diterrania invilando a personas del mundo del feminisopinión in el ámbito de la intervención socis acciones. lación directa la inequínca con el Trabajo Socia coma 作 tamiento de Barcelona llamado Canviem-ho? Este progra ma está dirigido a promover la concienciación, participa ción e implicación de hombres en la equidad de género. Se engloba dentro del SAH (Servicio de Atención al Hombre), un servicio ambulatorio dirigido a hombres que quieren cambiar su modelo de masculinidad para alejarse de pautas violentas (Margarida Sáiz, 2016). En Palma existe el Servei Municipal dAtenció integral a la Vilencia de Genere. Dentro de este hay 3 servicios específicos, relacions de parella no violentes, un servicio de atenció psicosocial dirigido a hombres que ejercen o han ejercdo violencia contra su pareja y quieren dejar de hacerlo (Ayuntamiento de Palma, 2020).

presente articulo no pretende ser, ni es, una recogda exhaustiva de todos los servicios dirigidos a hombres existentes en España. La intención es que sirva como punto de partida e incite a miembros de la profesión del Trabajo Social (y de todas las profesiones pertenecientes e nombrados para comprender cómo funcionan, cuál es u misión, etc. Ello puede contribuir a abrir una etapa de reivindicación profesional (y me atrevo a decir política), de demanda de apertura de servicios de estas características para abordar las desigualdades de género desde tro prisma, e intentar el cambio en hombres.

Existen algunas investigaciones que han analizado los movimientos de hombres en España. Pinilla (2012) encontró la confusión existente en torno a entender las España (no se ve la diferencia entre un grupo terapéutico de hombres sólo formado por hombres y un grupo de hombres igualitarios), o la falta de conocimiento por parte de los hombres de qué se hace en relación con el cuestionamiento de la masculinidad en otros lugares del territorio. Igualmente, se ha encontrado un perfil común en los grupos de hombres en funcionamiento, mostrano caracteristicas sociodemograficas comunes, que son: óvenes, autóctonos, con estudios superiores de la rama humanística y social (Marín, 2014), solteros, sin hijos, reconados con mujeres que trabajan en el ámbito públiho y que viven en grandes ciudades, que suelen sentirse 2002: Marín, 2014: Sanfélix, 2011). Defienden (Bovino, el de las mujeres, considerando la igualdad de género como un tema de hombres y de mujeres (Azpiazu, 2013: Pinilla, 2012; Sanfélix, 2011).

Todas las características anteriores nos permiten hacernos una idea de qué capas sociales tienen más difícil acceder a la posibilidad del cuestionamiento de la identidad de género. Personas con precariedad económica, por ejemplo, no tendrán la necesaria paz mental para tán cubiertas las necesidades básicas. Personas con poca formación / estudios, no podrán comprender y viajar hacia otras posiciones en el género si han vivido una socialización patriarcal que reproduce los estereotipo des... El Trabajo Social no puede perder estas realidades

3.5. Implicaciones para el Trabajo Social.

Cabe iniciar este espacio de reflexión con una de las afirmaciones más potentes existentes en el mundo de las trapolando esta afirmación al mundo (el Trat, 1970). Exconsidero que la práctica profesional, el toque persona que le damos, también es política. Si bien la visión y perspectiva de género y feminista parece estar bien arraigda (protección para mujeres que sufren violencia de gé nero, recursos, itinerarios laborales específicos, grupos de apoyo, etc.), no parece estarlo tanto la visión de los servicios públicos enfocados a atajar la raíz del problema. Como se ha ido dibujando durante los apartados anteriores, es hora para nosotros y nosotras, trabajadores y trabajadoras sociales, de comenzar a indicar el camino Para ello, se puede favorecer y trabajar para que acceda a servicios especializados como el programa Canviem-ho? (Barcelona). También se les puede ofrecer intervenciones interprofesionales con psicólogos o psicólogas. Y, ya desde nuestra propia reivindicación política (individual a traves de los colegios profesionales), reclamar que feminismo y la coeducación formen parte del itinerario curricular de todas las enseñanzas de este pals.

En la mayoría de los grados propios de la enseñanza de perspectiva de género como si sólo tuviera que ver co mujeres (Bakea Alonso, 2016). Además que ver con grados de Trabajo Social se imparten materias directa mente relacionadas con la perspectiva de género? En los que se imparten, ¿es una optativa o es de carácter obligatorio? Debemos empezar por exigirlo en nuestra propia profesión para luego expandirlo hacia otros terre nos. Como también debemos exigir, y lo apunta Alonso (2016) que los hombres sean beneficiarios de las políticas de igualdad, más alla de las politicas y programas destmuieres. $Y$ eso implica sumar presupuesto a las iniciativas, y escuchar a las mujeres para conocer cómo impacta la intervención con hombres en la vida de estas.

Es el proceso de transversalización del enfoque de género que debe involucrar una transformación en la agend del desarrollo en base a: diversidad, igualdad y eficacia. Solo asi comprenderemos las femineidades y las mascu linidades (Jorge García, 2003). Y no sólo esto, sino también debe abordare la forma. mismos hacia roles tradicionales (ser proveedor, trab jador incansable, etc), no estamos haciendo nada para cambiar el sistema de género ni para ofrecer nuevas visiones de la masculinidad. En cambio, si planteamos a estos hombres que quizás ellos deben cuidar a los hijos e hijas (si existen), y que es la mujer (en relaciones heterosexua les) quien puede trabajar, podemos esperar rechazo aceptación. En cualesquiera de los casos, seguramente conseguiremos una m
(2016) propone diversos ámbitos donde apuntar con una a) a) Empleo: trabajar con hombres desempleados pue de permitir que analicen su rol dentro de la familia y estereotipados.

b) Paternidad: los hombres tienen la oportunidad y el sus hijos e hijas. Es en el cuidado y la educación de proporcionen un modelo masculino interesado por las emociones, la igualdad con las mujeres y la corresponsabilidad en las tareas doméstica. (José Ángel Lozoya, 2012). Incidir desde el Trabajo Social en una nu va paternidad ya tendría efectos en la socialización de nuevas generaciones.

c) Mundo emocional: hay que rescatar las necesidades psicológicas de los hombres y desmontar las prohibiciones masculinas que impiden al hombre amar d) Sexualidad: desde el placer, reproducción, erotismo e identidad de género. España es el primer pás europeo en consumo de servicios de prostitución mayoritariamente entre la población masculina. Es necesaria la educación afectivo-sexual con los y las más jóvenes.

e) Salud: tanto en términos de autocuidados como en términos de hombres como cuidadores de otras personas. No pueden ser exentos de la sobrecarga que supone una distribución sexista de la responsabilidad la creencia que ellos son fuertes y no necesitan apoyo externo.

hombres hacia hombres. La violencia es una conducta aprendida, $\mathrm{y}$ como tal, puede o no-aprenderse o des prenderse... Difícil, pero no imposible.

os depende que la intervención se desarrolle en todos los ámbitos antes mencionados y otros que puedan quedar por proponer. Los trabajadores y las ponsabilidad y acción política. Debemuir de nuestra resparte de responsabilidad como aspecto del probuema las relaciones no equitativas entre mujeres $y$ hombres, así como comprometernos con el desarrollo de acciones políticas que proyecten nuestra posición no discrimina toria e intolerante ante la vigencia de la violencia de gé nero (García, 2003, p. 38).

í 2 décadas que siguen teHiendo plena valldez. ¡Tenemos deberes pendientes! Un buen ejemplo de este trabajo que la profesión debe ha que ofrecen alecicemos y participemos en formaciones Social que directomente apolan a nuevas formajo tervención con perspectiva de género (donde se englotemática de la masculinidad).

Oportunidades para profundizar en los aspectos teórcos que hemos ido planteando que, a su vez (y de ahí la de este articulo), deben estandarizarse, unifcasse y permitir la clarificación de conceptos, no su mezcla o utilización errónea. Digo esto porqué he podido de nuevas masculinidades, masculinidades alternativas 
(plural) y masculinidad alternativa (singular). Existen tambien, $y$ debe mantenerse una alerta desde disciplina del Trabajo Social, críticas constructivas que señalan el largo camino que aún queda por recorrer, dado que los movimientos profeministas llevan sólo 30 año organizándose en la sociedad civil (Ruiz, 2007). No s mos llegar a catalogar de "desempoderamiento" de lo hombres, para que se pongan a disposición del feminismo y se constituyan como auxiliar del mismo (Dupuis-De ri, 2008). También debe vigilarse el fenómeno del "post machismo", esa forma sutil de dominación que ejerce hombres con la perspectiva de la masculinidad cómplce (Luis Bonino, 2008). Hay que ayudar a los hombres a cuestionarse su expresión del género y evitar que se del hombre asesino, el monstruo (Pol Galofré, 2018). En ta que podría generar una ilusión de isualdad que no se corresponde con la realidad (Azpiazu, 2013. Bacete, 2017; Emakunde - Instituto Vasco de la Mujer, 2010).

\section{Conclusiones y propuestas.}

El presente artículo puede ser la puerta de entrada a un Trabajo Social a reflexionar y plantear teóricamente, de una forma más unificada, las intervenciones a hacer con perspectiva de género con hombres (haciéndolos partícipes) y escuchando la voz de las mujeres. Antes, pero, cabe destacar el conocimiento aportado en cuanto a clarificación de los conceptos así como el dibujo del perfil sociodemográfico atribuido al "hombre alternativo". Tenemos la misión de ampliar este perfil sociodemolos estamentos de la sociedad cuestionarse su género, ponerlo a prueba y cambiarlo por uno más integrador, comprensivo y que no implica la esclavitud (conductua y actitudinal).

Para ello, es preciso una formación adecuada para los trabajadores y las trabajadoras sociales. Desde forma ción obligatoria en materia de género en los grados, de intervención social con hombres. Pero no sólo eso. de intervención social con hombres. Pero no sólo eso. Debemos relindicar activa y politicamente que la co cer inherentes a los niveles formativos y académicos de nuestro país. Es indudable que la escuela forma parte, un porcentaje muy elevado, del proceso de socialización Una escuela inclusiva, con profesionales formados detectar y combatir los roles de género, permitirá a las futuras generaciones contar con herramientas de critica y cuestionamiento. Incluso, y en solidaridad con otra disciplina muy cuestionada en los últimos años por su supuesta irs: cvancia, debemos defender la fllosoria en las escuelas; espacios de reflexión, de diálogo con divers nal, insistir que no podemos ser agentes que profociola desigualdad de género. Los trabajadores y las trabajdoras sociales somo agentes de cambio. Debemos creénoslo y practicarlo. Debemos cuestionar los modelo viven por acompañar sus hijos e hijas a la escuela, por tener la casa en orden y por, además, visitar por ejemplo los servicios sociales porque no llegan a final de mes. Del mismo modo, debemos cuestionar los roles masculinos del trabajo por el trabajo, la ausencia de toda tarea doemociones o habrer con un o una profesionaly compartir problemas. Para hacer todo esto, debemos tener claro los conceptos básicos de género, igualdad, feminismo, masculinidad hegemónica, nuevas masculinidades, masculinidad alternativa, etc. Sin esto, no podremos entender el mundo en el que intervenimos ni podremos utilizar la empatía para entender a una mujer víctima de violencia de género, ni a un hombre que es agresor (de nuevo, entender, no justificar).

Qún came por recorrer, pero tengo la firme conviexperiencia, seguirá ampliando frentes y asumirá el reto profesional y político de seguir luchando por y para la igualdad de género, incorporando a los hombres como sujetos de cambio y sobre los que se puede, y se debe, intervenir.

5. Bibliografía.

HIGE (2018). Asociación de Hombres por la Igualdad de Género. Recuperado de http://ahige.org

onso, B. (2016). Trabajo social y perspectiva de género: los hombres como "colectivo" de intervención. (Coords), Respuestas Trandisciplinares en una So ciedad Global. Aportaciones desde el Trabajo Social. Logroño: Universidad de la Rioja. Recuperado en https://publicaciones.unirioja.es/catalogo/online/ CIFETS_2016/Monografia/pdf/TC031.pdf

Ayuntamiento de Palma (2020). Serveis d'atenció a la violencia de gènere. Recuperado de https://www. palma.cat/portal/PALMA/contenedori.jsp?sec-
cion $=s$ fdes d 4 v1.jsp\&contenido $=684038$ tipo $=6$ dResi $=1 \& \operatorname{cod} M e n u=839 \& \operatorname{cod} M e n u P N=1811 \& \operatorname{cod} A-$ directo $=2562$ \&language $=c a$

Azorín, C.M. (2017). Actitudes hacia la igualdad de género en una muestra de estudiantes de Murcia. Revista Complutense de Educación, 28(1), 45-60. https.// doi.org/10.5209/rev_RCED.2017.v28.4n1.48715

Azpiazu, J. (2013). Grupo de Hombres y discursos sobre la masculinidad: : inuevas configuraciones? Recuperado dos/informacion/gizonduz dokumentuak/es def/ adjuntos/jokin.azpizu.grupos.hombres.discursos. masculinidad.pdf

Azpiazu, J. (2017). Masculinidades y feminismo (1). BarceIona: Virus editorial y distribuidora, SCCL.

R. (2017). Nuevos hombres Buenos: Ia masculinidad en la era del feminismo (1). Barcelona: Ediciones

Badinter, E. (1993). XY: La identidad masculina. Madrid:

ergara, A., Riviere, J. y Bacete, R. (2008). Los hombres, la igualdad y las nuevas masculinidades. Vitoria-Gasperado de https://cpalsocial.org/documentos/892. $\mathrm{pdf}$

https://doi.org/10.1344/its.voii.32496
Bermúdez, M.M. (2013). Connell y el concepto de masculinidades hegemónicas: notas críticas desde la obra de Pierre Bourdieu. Estudios Feministas,
21 (1),283-300. https://doi.org/10.1590/S0104 21 (1), 283 - 300. https://doi.org/10.1590/S0104 $026 \times 2013000100015$

(a) el problema de la igualdad con las mujeres. En C., Lomas (Ed.) To-
dos los Hombres son iguales? Identidad masculina $y$ cambios sociales (p. 105-144). Barcelona: Paidós y cambios sociales (p. 105-144). Barcelona: Paidós.
Recuperado de http://www.bizkaia.eus//Homez/ Archivos/DPTO1/Temas/Pdf/ca luisbonino pdf?has-

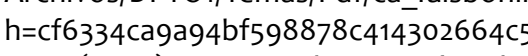

Bonino, L. (2008). Micromachismos: el poder masculino en la pareja "moderna". En C. Espada, (Ed.), Voces de Hombres por la igualdad (p. 89-109). Recuper do de htps.//Vocesdehombres.tles.wordpress. no-en-2008/07/micromachistejo-modernas

Carabí, A. y Armengol, J.M. (2015). Masculinidades alternativas en el mundo de hoy. Barcelona: Icaria editorial S.A.

Chouciño, S. (2017). Pas de la Masculinitat Hegemònica a les Noves masculinitats: evolució de les masculinitats des de la mirada de les dones [Trabajo de Fin de Grado, Universitat de Barcelona]. Dipòsit Digital de Universitat de Barcelona. http://diposit.ub.edu/d pace/handle/2445/114954

Connell, R. (1995). La organización social de la masculinidad. Berkeley: University of California Press.

ge. Masculinities and Social Change, 1(1), 4-18. http dx.doi.org/10.4471/mcs.2012.01

Connell, R.W., Hearn, J. \& Kimmel, M.S. (2005). Handbook of Studies on Men \& Masculinities. Thousan Oaks: Sage Publications, Inc.

Connell, R.W. \& Messerschmidt, J. (2005). Hegemo nic Masculinity: rethinking the Concept. Gen-
der and Society, 19 (6), 829-859. https://doi. org/10.1177//0891243205278639

de Keijzer, B. (2003). Hasta donde el cuerpo aguante Género, cuerpo y salud Masculina. En: C., Cáceres perspectivas y propuestas desde América Latina (p. 137-152). Perú: Universidad Peruana Cayetano $\mathrm{He}$ redia. Recuperado de http://agendadelasmujeres. com.ar/pdf/est_masc_01.pd

Dupuis-Déri, F. (2008). Les homes profeministes: compagnons de route ou faux amis? Recherces feminis tes, $21(1), 149-169$. htps.//doi.org/10.7202/018314ar

linidades e igualdad: Análisis multidisciplinar. Viuria-Casteiz: Emakunde - Instituto Vasco de la Muier. rnández, A. (2009). La construcció social de les masculnitats igualitàries: Quan no tots els homes són igual [Trabajo de Fin de Máster no publicado]. Univers tat de Barcelona.

Flecha, R., Puigvert, L. \& Ríos, O. (2013). The New Alter natives Masculinities and the Overcoming of Gender Violence. International and Multidisciplinary Journal of Social Sciences, 2 (1), 88-113. http://dx.do
org//10.4471/rimcis.2013.14 Gabarró, D. (2011). Transformar a los Hombres: un reto social. Barcelona: QSM.

Cabbard, K. (2008). Homes de pel-lícula. En: A., Carabi J.M., Armengol (Ed.). La masculinidad a debate (

Galofré, P. (2018). Despatologitzem el col-lectiu LGTBI+. Hèlia Dones i SEPC (Ed.). II Congrés per a l'eradicació sanitaris. Barcelona.

García, J. (2003). ¿Y los varones? Trabajo social, género y masculinidades. Revista perspectivas: notas sobre intervención y acción social, 8 (13), 29-41. https://doi. org/10.29344/07171714.13.1199

Garousi, S., Khajeh, R. \& Garrusi, B. (2016). Concerns abouy body change behaviors, and construction of flexible masculinity among Iranian students. Quality \& Quantity, 56 (6), 2831-2846. https://do org//10.1007/511135-016-0448

. (2003). Men and masculinities. Buckingham: Open University Press. der equality. Sweden: Save the Children.

Kaufman, M. (1999). Las siete p's de la violencia de los Hombres. International Association for Studies of Men, 6(2), 6-9. Recuperado de http://www.mchaelkaufman.com/wp-content/uploads/2009/0 kaufman-las-siete-ps-de-la-violencia-de-los-hom bres-spanish.pdf

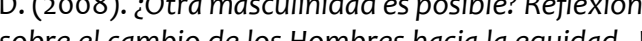
rez: Ayunamion

D, Szil, P, Lozoya, JA, y

y Bonino, L. (2010). Algunas sugerencias para impulsar grupos de hombres
igualitarios. Recuperado de http://www.jerez.es fileadmin/Documentos/hombresxigualdad/fondo documental/Movimiento_hombres_igualitarios/Su-

gerenciasimpulsargruposdehombres.pdf
zoya, J.A. (2012). El papel de los hombres por la igualdad. Recuperado de http://www.emakunde.euskad.

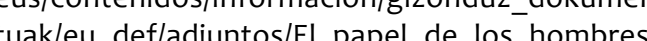
por_la_igualdad.pdf

Marín, A. (2014). Veus cap a la igualtat: Anàlisi de processos de construcció de masculinitats plurals [Trabajo de Fin de Grado, Universitat de Barcelona]. Dipòsit Digital de la Universitat de Barcelona. http://diposit. ub.edu/dspace/handle/2445/65980

Millett, K. (1970). Política Sexual. New York: Doubleday. com a alternatives a la violència. Dossiers feministes, (1), 201-223. hit. der role conflicte research: New conceptual models and research paradigms. En: J. Wong, \& S. Wester (Ed.), APA Handbook of Men and Masculinities (p. 51 80). Washington, DC: APA.

'Neil, J., Denke, R. \& Blazina, C. (2016). Gender Role Conflict Theory, Research, and Practice: Implcations for understanding the Human - Animal Bond. En: B., Christopher \& L. R., Kogan (Ed.). Men Friend. (p. 11-45). Switzerland: Springer. htp doi.org/10.1007/978-3-319-30097-9_2 
Peña, J. C. (2013). Construcción de masculinidades igualitarias atractivas: la transmisión de valores a través de actos comunicativos en contextos [Tesis doctoral, Universitat de Barcelona]. Dipòsit Digital de la Universitat de Barcelona. http://diposit.ub.edu/dspace/ bitstream/2445/48500/1/JCPA_TESIS.pdf

Pheterson, G. (2013). Mujeres en flagrante delito de independencia. Barcelona: Edicions Bellaterra.

Pinilla, D. (2012). Masculinidades: Un acercamiento a los grupos de hombres por la igualdad en el Estado español. [Tesis de Máster, Universidad de Zaragoza]. https://www.joaquimmontaner.net/Saco/tesina\%20 final\%20master\%20masculinidades\%20un\%20acercamiento.pdf

Ricardo, C. (2015). Masculinidades y cambios en el poder: un documento de debate sobre la participación de los hombres en la igualdad de género desde Beijing 1995 hasta el año 2015. MenEngage, ONU Mujeres y UNFPA.

Ríos, O. (2013). Homes en diàleg: noves masculinitats alternatives. Prevenint la violència de gènere a través de l'educació. Àmbits de psicopedagogia, 39 (1), 17-23. Recuperado de http://ambitsaaf.cat/article/ view/821

Rodrigo, B. (2018). Los hombres que se rindieron al feminismo: "ser machirulos nos vuelve tóxicos". El Confidencial. https://www.elconfidencial.com/espana/2018-03-07/hombres-huelga-8marzo-feminismo-masculinidad-toxica_1530852/

Ruiz, A. (2007). Masculinidad/es y movimientos de Hombres. Málaga: AHIGE.

Sáiz, M. (2016). Recerca, polítiques públiques i serveis socials. En: N., Roca, C., Porrúa, M., Yepes, M., Martínez, N., Codina, A., Fernández i S., Ruiz. Recuperació de les dones en situació de violència masclista de parella: descripció i instrumentació (p. 8-27). Barcelona: Ajuntament de Barcelona.

Salas, J.M. y Campos, A. (2001). Masculinidad en el nuevo milenio. [Ponencia]. I Encuentro centroamericano acerca de las masculinidades. San José. Recuperado de http://menengage.org/wp-content/ uploads/2014/06/Masculinidad_nuevo_milenio.pdf

Sanfélix, J. (2011). Las nuevas masculinidadēes. Los hombres frente al cambio en las mujeres. Prisma Social 1 (7), 220-247. Recuperado de http://www.redalyc. org/articulo.oa?id=353744579008

Schongut, N. (2012). La construcción social de la masculinidad: Poder, hegemonía y violencia. Psicología, Conocimiento y Sociedad, 2 (2), 27-65. Recuperado de http://www.redalyc.org/pdf/4758/475847408003. pdf

Serrano, T. y Schirralli, M. (2009). Hacia una nueva masculinidad: reeducando en el desarrollo de nuevos modelos de masculinidad. Castelló: Patim Castellón. Recuperado de http://www.asecedi.org/nueva/ masculinidades/UNIDAD\%20DIDACTICA\%20DEFINITIVA.pdf

Varela, N. (2008). Feminismo para principiantes. Barcelona: B.S.A.

Wong, J. \& Horn, A. (2016). Enhancing and diversifying research methods in the Psychology of men and masculinities. En J. Wong \& S. Wester (Ed.), APA
Handbook of Men and Masculinities (p. 231-255). Washington, DC: APA. 